\section{Arbeitskreis Technikfolgenab- schätzung und -bewertung des Landes Nordrhein-Westfalen - AKTAB NRW}

\author{
von Robert Tschiedel, AKTAB NRW
}

\begin{abstract}
Der Arbeitskreis Technikfolgenabschätzung und -bewertung des Landes NordrheinWestfalen (AKTAB NRW) ist ein 1992/93 auf Initiative des Wissenschaftsministeriums NRW entstandener interdisziplinärer Arbeitskreis von Vertretern v.a. aus Wissenschaft, Wirtschaft, Verwaltung und weiterer Öffentlichkeit, die sich in Forschung und Praxis regelmäßig mit gesellschaftlichen und dabei v.a. technischen Innovationen, ihren Folgen, ihrer Bewertung und ihrer Gestaltung auseinandersetzen.
\end{abstract}

Der Arbeitskreis Technikfolgenabschätzung und -bewertung des Landes Nordrhein-Westfalen knüpft an das vorausgegangene Programm "Mensch und Technik - sozialverträgliche Technikgestaltung" an, das die Landesregierung zur Begleitung des technologiepolitisch geförderten Strukturwandels in NRW ins Leben gerufen hatte, an die Arbeit der 1987 eingesetzten Kommission "Mensch und Technik" sowie an den von 1990 bis 1995 aktiven Landtagsausschuß "Mensch und Technik".

Er versteht sich als dezentrales Netzwerk (im Unterschied zu institutionellen Lösungen) mit dem Ziel, an der umwelt- und sozialverträglichen Gestaltung zukunftsrelevanter Innovationen mitzuwirken und dazu insbesondere auch einen breiten gesellschaftlichen Diskurs über (technische) Innovationen zu initiieren und moderierend zu begleiten.

Der AKTAB besteht derzeit aus 21 Mitgliedern und einem Wirtschaftsbeirat.

\section{Aufgaben und Tätigkeiten}

Als Fachgremium steht der AKTAB zur Verfügung als Ansprechpartner

- für Politikberatung und Entscheidungsvorbereitung des Landtages und der Landesregierung,
- für Innovationsberatung und -begleitung in Wirtschaftsunternehmen,

- zur Begutachtung von Förderanträgen sowohl für Antragsteller als auch für Fördereinrichtungen,

- zur Moderation und Mediation von landespolitisch relevanten Innovationsprozessen.

Der AKTAB versteht sich darüber hinaus als

- Basisnetzwerk für TA-Projekte in NRW und

- Netzknoten für bundesweite und internationale Kooperationen sowie als Ort für Koordination, Dokumentation, Information und Öffentlichkeitsarbeit.

Seine inhaltliche Arbeit erfolgt durch Projektarbeit seiner Mitglieder (möglichst in Verbünden) $\mathrm{zu}$

- Querschnittsthemen wie Theorieentwicklung, Methodenentwicklung, TA in der Lehre und Wertewandel sowie durch

- Modellprojekte zu ausgewählten Schwerpunktthemen.

Durchgeführt wurden und werden neben Dokumentationen zur TA in NRW Projekte u.a. zu den Themen Mobilität, Bio- und Gentechnik, Methoden der TA. Ein umfangreiches Handbuch zur Innovationsorientierten Technikfolgenabschätzung, Technikbewertung und -gestaltung (ITA) erscheint im Frühjahr 1999. Auf einer Tagung im Dezember 1998 werden weitere Themenbereiche und Projekte erarbeitet.

Ein wesentlicher Schwerpunkt der Bemühungen des AKTAB sind "Diskurse zur industriellen TA", die mit Wirtschaftsvertretern stattfinden und dazu führen sollen, ITA stärker als bisher für die Mitgestaltung auch betrieblicher Innovationsprozesse zu qualifizieren.

Der AKTAB bemüht sich um die Einwerbung von Fördermitteln und unterstützt seine Mitglieder dabei.

Zur Koordination der Arbeit, zum Austausch von Forschungsergebnissen, Informationen und Meinungen sowie zur Projektentwicklung, Projektberatung und Projektbewertung treffen sich die Mitglieder des AKTAB ca. viermal jährlich zu einer Sitzung. 
Der AKTAB führt in unregelmäßigen Abständen Veranstaltungen für die allgemeine und die Fachöffentlichkeit durch.

\section{Formalia}

Neue Mitglieder werden auf Vorschlag eines Mitglieds durch Kooptation mit einfacher Mehrheit berufen. Dabei strebt der AKTAB interdisziplinäre und geschlechterbezogene Ausgewogenheit an.

Die Mitglieder des AKTAB wählen für je zwei Jahre ein Mitglied mit einfacher Mehrheit zur Sprecherin/zum Sprecher. Sie/er wird von der Vorgängerin/vom Vorgänger vertreten. Der AKTAB kann einen Vorstand bestimmen.

Der AKTAB hat eine Geschäftsstelle, die am Standort der Sprecherin/des Sprechers angesiedelt ist.

Zur Begutachtung von Projektanträgen und für ähnliche Aufgaben kann der AKTAB ein Gutachtergremium zur Vorbereitung des Votums des AKTAB durch Wahl bestimmen.

Zur Förderung der Zusammenarbeit mit der Wirtschaft hat der AKTAB einen Wirtschaftsbeirat, der von einem Mitglied des AKTAB betreut wird. Der AKTAB kann weitere Gremien bestellen.

\section{Mitglieder des AKTAB NRW}

Ministerium für Schule, Weiterbildung, Wissenschaft und Forschung des Landes NRW

Dr. Wolf Jenkner

Ministerium für Arbeit, Soziales und Stadtentwicklung, Kultur und Sport des Landes NRW

Evamaria Küppers-Ulrich

Bundesministerium für Bildung, Wissenschaft, Forschung und Technologie

Dr. Hach

RWTH Aachen, Hochschuldidaktisches Zentrum / Lehrstuhl Informatik im Maschinenbau

Prof. Dr.-Ing. Klaus Henning

RWTH Aachen, Institut für Stadtbauwesen

Prof. Dr.-Ing. Klaus J. Beckmann

Universität -GH-Essen, Fachbereich Philosophie

Prof. Dr. Carl Friedrich Gethmann

Universität Bielefeld, Institut für Wissenschafts- und

Technikforschung

Prof. Dr. Wolfgang Krohn

Universität Bielefeld, Lehrstuhl für Genetik, Fakultät für Biologie

Prof. Dr. Alfred Pühler
Universität Dortmund, Wirtschafts-und Sozialwissenschaften

Prof. Dr. Hartmut Neuendorff

Universität Dortmund, Lehrstuhl Technik und Gesellschaft

Prof. Dr. H. Hirsch-Kreinsen

Ruhr-Universität Bochum, Institut für Arbeitswissenschaften

Dr.-Ing. Rainer Skrotzki

FernUniversität Hagen, Fachbereich Erziehungs-, Sozial- und Geisteswissenschaften, Lehrgebiet Internationale Politik - Vergleichende Politikwissenschaft

Prof. Dr. Gerd Simonis

Bergische Universität - GH-Wuppertal, Forschungsinstitut für Telekommunikation

Prof. Dr. Reinhard Rock

Wissenschaftszentrum NRW, Institut Arbeit und Technik, Produktionssysteme

Dr.-Ing. Peter Brödner

Wissenschaftszentrum NRW

Dr. Dirk Matejovski

Sekretariat für Zukunftsforschung

Prof. Dr. Rolf Kreibich

Mensch, Arbeit \& Technik, Sell \& Partner GmbH

Dr. Paul Fuchs-Frohnhofen

Bayer AG, Zentrale Technik, Technische Entwicklung

Dr. Rüsseler

Deutsche Forschungsanstalt für Luft- und Raumfahrt e.V., Hauptabteilung Verkehrsforschung

Dr. Hans-Gustav Nüßer

Landesinstitut Sozialforschungsstelle

Dr. Gerd Peter

TaT Transferzentrum für angepaßte Technologien $\mathrm{GmbH}$, Institut für Technik und Gesellschaft

Prof. Dr. Robert Tschiedel

Rhein-Ruhr-Institut für Sozialforschung und Politikberatung

Prof. Dr. Heribert Schatz

Klaus Steilman Institut für Innovation $\mathrm{GmbH}$

Prof. Dr. Wolf D. Hartmann

\section{Mitglieder des Wirtschaftsbeirates}

Waggonfabrik Talbot GmbH \& Co. KG

Dr.-Ing. Klaus Altenburg

Jobo Labortechnik GmbH u. Co. KG

Dipl.-Ing. Rainer Bernhardt

Handwerkskammer Aachen

Dipl.-Volksw. Otto Brink

agiplan $A G$

Dr.-Ing. Reinhard Ehl

Gerling-Konzern Münster GmbH

Dr. Frank Grund

Windel Textil GmbH \& Co

Dipl.-Kfm. Christian Meyer-Stork 
Franz Zentis GmbH \& Co

Prof. Dr. Hans-Jörg Raeuber

Bayer $A G$

Dr. Wolfgang Rüssler

Schmidt + Clemens $\mathrm{GmbH}+\mathrm{Co}$

Dipl.-Ing. Christoph Schmidt-Krayer

Technologieberatungsstelle beim DGB Landesbezirk NRW e.V.

Dr. Ulrich Weber TBS

\section{Sprecher}

Prof. Dr. Robert Tschiedel

\section{Geschäftsstelle}

TaT Transferzentrum für angepaßte

Technologien $\mathrm{GmbH}$

Hovesaatstraße 6, D-48432 Rheine

Tel.: ++ 49 (0) 5971/990-0

Fax: ++ 49 (0) 5971/990-150

E-mail: tatgermany@aol.com

Internet: http://members.aol.com/tatgermany

Dr. Thomas Becker, Tel.: 05971/990-111

Kirsten Tacke M.A., Tel.: 05971/990-113

\section{Veröffentlichungen des AKTAB NRW}

Bröchler, Stefan (1997): Politische Technikfolgenabschätzung in Nordrhein-Westfalen. In: R. Graf v. Westphalen (Hrsg.), Technikfolgenabschätzung. Oldenbourg, S. 388-409.

Bröchler, Stefan; Simonis, Georg (Hrsg.) (1996): Perspektiven der Technikfolgenabschätzung und -gestaltung in Nordrhein-Westfalen. Schriftenreihe des Arbeitskreises Technikfolgenabschätzung und -bewertung des Landes NRW, Heft 1.

Ministerium für Wissenschaft und Forschung des Landes NRW (Hrsg.) (1995): Technikfolgen - Forschung Dokumentation 1995. Düsseldorf.

FernUniversität Hagen, Fachbereich ESGW, Lehrgebiet Internationale Politik/Vergleichende Politikwissenschaft (Hrsg.): VITA-Newsletter (bisher erschienen: 1 und 2/97). Hagen.

\section{TA-Netz für Brandenburg}

\author{
von Peter Ackermann, Brandenburgisches \\ Umweltforschungszentrum e.V.
}

TA wird im Land Brandenburg als wichtiges Element für die Gestaltung einer Technologiepolitik im Sinne der nachhaltigen Entwicklung verstanden. Unter den gegenwärtigen Bedingungen (Mittelknappheit) erfolgt die Umsetzung mittels schwerpunktorientierter befristeter TA-Projekte. Um die vorhandenen TA-Potentiale zu bündeln, haben sich auf Initiative des Brandenburgischen Umweltforschungszentrums (BUFZ) die TAAkteure in Brandenburg zu einem Arbeitskreis zusammengeschlossen.

Das Leitbild einer nachhaltig zukunftsverträglichen Entwicklung heißt, die zentralen Entwicklungsparameter von Mensch und Umwelt so zu gestalten, daß eine dauerhafte Existenzfähigkeit der menschlichen Gesellschaft gewährleistet ist (Kreibich 1996, S.32). Daraus ergibt sich die Zielsetzung, gleichzeitig die natürlichen Lebensgrundlagen zu erhalten, wirtschaftlichen Wohlstand $\mathrm{zu}$ ermöglichen und für soziale Gerechtigkeit zu sorgen (Zieldreieck). Für die Wissenschaft der Zukunft (und damit auch für Forschung und Technologieentwicklung) bedeutet dies (Staufenbiel 1996, S. 224):

- Wissenschaft muß als Kollektivunternehmen agieren. Die starken Wechselwirkungen zwischen den technischen und den sozialen Subsystemen und die Komplexität der Probleme erfordern eine gemeinsame Anstrengung.

- Das Kollektivunternehmen Wissenschaft der Zukunft erfordert institutionelle Formen, ein Ernst nehmen seitens der Politik und einen direkten Zugang zu den gesellschaftlichen Kommunikationssträngen.

Damit wird die Steuerung von Technikentwicklung als ein entscheidendes Feld einer nachhaltig zukunftsverträglichen Entwicklung nicht mehr nur eine Aufgabe von Politik und Markt, sondern wird durch die gesamte Gesellschaft vorgenommen und verantwortet. Und auch die entstehenden Lasten trägt die gesamte Gesellschaft verbindlich. Das wäre ein wichti- 